\title{
A FOURTH-ORDER EQUATION WITH CRITICAL GROWTH: THE EFFECT OF THE DOMAIN TOPOLOGY
}

\author{
Jéssyca Lange Ferreira Melo - Ederson Moreira dos Santos
}

ABSTRACT. In this paper we prove the existence of multiple classical solutions for the fourth-order problem

$$
\begin{cases}\Delta^{2} u=\mu u+u^{2 *-1} & \text { in } \Omega, \\ u, \quad-\Delta u>0 & \text { in } \Omega, \\ u, \quad \Delta u=0 & \text { on } \partial \Omega,\end{cases}
$$

where $\Omega$ is a smooth bounded domain in $\mathbb{R}^{N}, N \geq 8,2_{*}=2 N /(N-4)$ and $\mu_{1}(\Omega)$ is the first eigenvalue of $\Delta^{2}$ in $H^{2}(\Omega) \cap H_{0}^{1}(\Omega)$. We prove that there exists $0<\bar{\mu}<\mu_{1}(\Omega)$ such that, for each $0<\mu<\bar{\mu}$, the problem has at least $\operatorname{cat}_{\Omega}(\Omega)$ solutions.

\section{Introduction}

Brézis and Nirenberg [8] investigated the question about the existence of a classical solution for the second-order problem

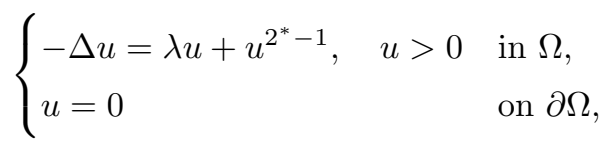

2010 Mathematics Subject Classification. Primary 35J30; Secondary 35B33, 58E05.

Key words and phrases. Biharmonic equation, Critical exponent, Lusternik-Schnirelman category, Positive solutions..

J.L.F. Melo was supported by FAPESP \#2010/00603-9 grant.

E. Moreira dos Santos was partially supported by CNPq \#309291/2012-7 grant and FAPESP \#2010/19320-7-2 grant. 
where $2^{*}=2 N /(N-2), N \geq 3$ and $\Omega \subset \mathbb{R}^{N}$ is a bounded smooth domain. Let $\lambda_{1}(\Omega)$ be the first eigenvalue of $\left(-\Delta, H_{0}^{1}(\Omega)\right)$. It was proved in [8] that:

(a) (BN) has no solution for $\lambda \geq \lambda_{1}(\Omega)$. If $\Omega$ is also starshaped, then the Pohožaev identity [22] guarantees that (BN) has no solution for $\lambda \leq 0$.

(b) For $N \geq 4$ the problem (BN) has a solution for every $0<\lambda<\lambda_{1}(\Omega)$.

(c) In case $N=3$, also called the critical dimensional case, the problem is more complex. Indeed, in case $\Omega$ is starshaped, (BN) has no solution when the parameter $\lambda$ is positive and small enough and, in the particular case when $\Omega$ is an open ball, (BN) has a solution if, and only if, $\lambda_{1}(\Omega) / 4<$ $\lambda<\lambda_{1}(\Omega)$.

In contrast to the case when $\Omega$ is starshaped, consider $N \geq 3$ and a ring $\Omega \subset$ $\mathbb{R}^{N}$. We know that the embedding $H_{0, \text { rad }}^{1}(\Omega) \hookrightarrow L^{2^{*}}(\Omega)$ is compact; see Ni [21, Radial Lemma]. Hence, (BN) has a radial solution for every $\lambda \in\left(-\infty, \lambda_{1}(\Omega)\right)$.

The above description shows that the shape of $\Omega$ and the dimension $N$ interfere in the set of solutions for (BN). Rey [23], [25] observed that the number of solutions of (BN) is strongly influenced by the topology of $\Omega$. Indeed, using arguments based on the Lusternik-Schnirelman category, it was proved by Rey [23] for $N \geq 5$, after by Lazzo [17] for $N \geq 4$, that (BN) has at least $\operatorname{cat}_{\Omega}(\Omega)$ solutions if the parameter $\lambda>0$ is sufficiently small.

When using the Lusternik-Schnirelman theory to get the existence of multiple solutions for the problem (BN), the topological arguments applied require that $\lambda$ be positive and close to zero. In particular, such procedure only works for non-critical dimensions.

In this paper, also inspired by the just described results, we study the existence of multiple classical solutions for the fourth-order problem

$$
\begin{cases}\Delta^{2} u=\mu u+u^{2_{*}-1} & \text { in } \Omega, \\ u, \quad-\Delta u>0 & \text { in } \Omega, \\ u, \quad \Delta u=0 & \text { on } \partial \Omega,\end{cases}
$$

where $\Omega$ is a smooth bounded domain in $\mathbb{R}^{N}, N \geq 8,0<\mu<\mu_{1}(\Omega), \mu_{1}(\Omega)$ is the first eigenvalue of $\left(\Delta^{2}, E(\Omega)\right), E(\Omega):=H^{2}(\Omega) \cap H_{0}^{1}(\Omega)$, and $2_{*}=2 N /(N-4)$ is the critical exponent for the embedding of $E(\Omega)$ into $L^{2 *}(\Omega)$.

In [27], van der Vorst proved that if $N \geq 5, \mu \geq \mu_{1}(\Omega)$ or, $\mu \leq 0$ and if the domain $\Omega$ is starshaped, then $(\mathrm{P})$ has no solution. In the same paper, assuming that $\Omega$ is a general bounded regular domain in $\mathbb{R}^{N}, N \geq 8$ and $\mu \in\left(0, \mu_{1}(\Omega)\right)$, it was proved that $(\mathrm{P})$ has a solution. Later, Gazzola et al. [13] proved that $N=5,6,7$ are the critical dimensions for the problem (P) in the sense that (P) has no solution if $\mu>0$ is small enough and $\Omega$ is an open ball in $\mathbb{R}^{N}$.

Our main contribution in this paper is to present a result on the existence of multiple solutions for (P) for all non-critical dimensions, namely, for all $N \geq 8$. 
THEOREM 1.1. If $\Omega$ is a smooth bounded domain in $\mathbb{R}^{N}, N \geq 8$, then there exists $0<\bar{\mu}<\mu_{1}(\Omega)$ such that, for each $0<\mu<\bar{\mu}$, the problem $(\mathrm{P})$ has at least $\operatorname{cat}_{\Omega}(\Omega)$ classical solutions.

We mention that El-Mehdi and Selmi [11], inspired by the procedures adopted in [23]-[25] to deal with $(\mathrm{BN})$, proved that for $N>8$ the problem (P) has at least $\operatorname{cat}_{\Omega}(\Omega)$ solutions if the parameter $\mu>0$ is sufficiently small.

More recently, Abdelhedi [1] used similar techniques to those in [11] to prove the existence of multiple solutions for a similar problem.

We stress that the condition $N>8$ seems essential in the arguments in [1] and [11] as well as $N \geq 5$ was required by Rey in [23]. In particular, it has been left as open problem the influence of the domain topology on the existence of multiple solutions for problem (P) in case $N=8$; see [11, Remark 1.4].

To prove our result we use a different approach from that in $[1,11]$, which seems more direct and works for $N \geq 8$. We must also say that instead of projections we employ suitable extensions; for instance compare [11, p. 419] and (4.3) in this paper. In addition, we believe that the extension and symmetrization techniques in this paper for functions in $H^{2}(\Omega) \cap H_{0}^{1}(\Omega)$ will be useful to treat other fourth-order problems. In particular, the proofs of Lemmas 4.4, 4.6 and equation (4.9) exemplify how our extension procedure replaces the standard extension by zero used to deal with second-order problems.

This manuscript is organized as follows. In Section 2 we set the variational framework. In Section 3 we prove some compactness results and then we prove Theorem 1.1 in Section 4. We also include an appendix within we prove some technical results from Sections 3 and 4 .

\section{Variational framework}

We first fix some notations. We consider the space $E(\Omega):=H^{2}(\Omega) \cap H_{0}^{1}(\Omega)$ endowed with the norm $\|u\|:=|\Delta u|_{2}$, induced by the inner product

$$
\langle u, v\rangle=\int_{\Omega} \Delta u \Delta v d x, \quad u, v \in E(\Omega) .
$$

In this part we will consider the following general assumptions: $\Omega \subset \mathbb{R}^{N}$, $N \geq 5$, is a bounded smooth domain and

$$
0<\mu<\mu_{1}(\Omega)=\inf _{\substack{u \in E(\Omega) \\ u \neq 0}} \frac{|\Delta u|_{2}^{2}}{|u|_{2}^{2}}=\inf _{\substack{u \in E(\Omega) \\|u|_{2}=1}}|\Delta u|_{2}^{2}
$$

Consider the Sobolev constant for the embedding $E(\Omega) \hookrightarrow L^{2 *}(\Omega)$, given by

$$
S(\Omega)=\inf \left\{\int_{\Omega}|\Delta u|^{2} d x: u \in E(\Omega), \int_{\Omega}|u|^{2 *} d x=1\right\} .
$$


It is known that $S(\Omega)$ does not depend on $\Omega$ and $S(\Omega)$ is not achieved except when $\Omega=\mathbb{R}^{N}[26]$. Moreover, $S(\Omega)=S$, where

$$
S=\inf \left\{\int_{\mathbb{R}^{N}}|\Delta u|^{2} d x: u \in \mathcal{D}^{2,2}\left(\mathbb{R}^{N}\right), \int_{\mathbb{R}^{N}}|u|^{2_{*}} d x=1\right\},
$$

which is attained precisely by the functions $S^{(4-N) / 8} \varphi_{\delta, a}$, with

$$
\begin{aligned}
\varphi_{\delta, a}(x)=\frac{[(N-4)(N-2) N(N+2)]^{(N-4) / 8} \delta^{(N-4) / 2}}{\left(\delta^{2}+|x-a|^{2}\right)^{(N-4) / 2}} & \\
= & \frac{C_{N} \delta^{(N-4) / 2}}{\left(\delta^{2}+|x-a|^{2}\right)^{(N-4) / 2}},
\end{aligned}
$$

for varying $a \in \mathbb{R}^{N}$ and $\delta>0[13$, Lemma 1$]$. We recall that the functions given by (2.3) are precisely the positive regular solutions of

$$
\Delta^{2} u=u^{2 *-1} \text { in } \mathbb{R}^{N} .
$$

Define, for $\mu \in\left(0, \mu_{1}(\Omega)\right)$, the norm

$$
\|u\|_{\mu}:=\left(|\Delta u|_{2}^{2}-\mu|u|_{2}^{2}\right)^{1 / 2}, \quad \text { for all } u \in E(\Omega),
$$

and observe the equivalence

$$
\|u\|_{\mu} \leq\|u\| \leq c(\Omega)\|u\|_{\mu}, \quad \text { for all } u \in E(\Omega),
$$

where $c(\Omega)=\left(1-\mu /\left(\mu_{1}(\Omega)\right)\right)^{-1 / 2}>0$.

To study the existence of solutions for the problem (P), we will consider the functional

$$
I(u):=\frac{1}{2} \int_{\Omega}|\Delta u|^{2} d x-\frac{\mu}{2} \int_{\Omega}\left(u^{+}\right)^{2} d x-\frac{1}{2_{*}} \int_{\Omega}\left(u^{+}\right)^{2_{*}} d x, \quad u \in E(\Omega) .
$$

Definition 2.1. Let $\Omega \subset \mathbb{R}^{N}, N \geq 8$, be a bounded smooth domain and $0<\mu<\mu_{1}(\Omega)$. We say that $u \in E(\Omega)$ is a weak solution of $(\mathrm{P})$ if $u$ is a critical point of $I$, that is, $u \in E(\Omega)$ satisfies

$$
\int_{\Omega} \Delta u \Delta v d x=\mu \int_{\Omega}\left(u^{+}\right) v d x+\int_{\Omega}\left(u^{+}\right)^{2_{*}-1} v d x, \quad \text { for all } v \in E(\Omega) .
$$

Lemma 2.2. Let $\Omega \subset \mathbb{R}^{N}, N \geq 8$, be a bounded smooth domain and $0<\mu<$ $\mu_{1}(\Omega)$. Then the $C^{4}(\bar{\Omega})$-classical solutions of $(\mathrm{P})$ are precisely the nontrivial critical points of the functional I defined by (2.6).

Proof. The results in [26, Appendix B], [2, Theorem 12.7] and [14, Theorems 2.19 and 2.20] guarantee that the nontrivial critical points of $I$ are precisely the classical solutions of $(\mathrm{P})$. We mention that the arguments in $[9, \mathrm{p} .375]$ can be used to prove that every nontrivial critical point of $I$ satisfies $u,-\Delta u>0$ in $\Omega$. 
From now on we will turn our attention to study the functional $I$, or equivalently to study

$$
I_{\mu}(u):=\int_{\Omega}|\Delta u|^{2} d x-\mu \int_{\Omega}\left(u^{+}\right)^{2} d x
$$

restricted to the manifold

$$
V:=\{u \in E(\Omega): \psi(u)=1\} \quad \text { where } \psi(u):=\int_{\Omega}\left(u^{+}\right)^{2 *} d x .
$$

We also define

$$
m(\mu, \Omega):=\inf \left\{I_{\mu}(u) ; u \in V\right\}
$$

and, if $\Omega=B_{\rho}(0)$, we denote $m(\mu, \rho):=m\left(\mu, B_{\rho}(0)\right)$.

We will prove that the functional $\left.I_{\mu}\right|_{V}$ has at least as many critical points as the Lusternik-Schnirelman category of $\Omega$, which up to suitable multiplicatives constants are classical solutions for $(\mathrm{P})$.

\section{Compactness}

The next lemma describes the lack of compactness of the embedding of $\mathcal{D}^{2,2}\left(\mathbb{R}^{N}\right)$ into $L^{2 *}\left(\mathbb{R}^{N}\right)$. A similar result for the embedding of $\mathcal{D}^{1,2}\left(\mathbb{R}^{N}\right)$ into $L^{2^{*}}\left(\mathbb{R}^{N}\right)$ is proved in [28, Lemma 1.40]; see also [4], [5], [18].

LEMma 3.1 (Concentration and compactness). Let $\left(u_{n}\right) \subset \mathcal{D}^{2,2}\left(\mathbb{R}^{N}\right)$ be a sequence such that

$$
\begin{aligned}
u_{n} \rightarrow u & \text { in } \mathcal{D}^{2,2}\left(\mathbb{R}^{N}\right), \\
\left|\Delta\left(u_{n}-u\right)\right|^{2} \stackrel{*}{\rightarrow} \lambda & \text { in the sense of measures on } \mathbb{R}^{N}, \\
\left|u_{n}-u\right|^{2 *} \stackrel{*}{\rightarrow} \nu & \text { in the sense of measures on } \mathbb{R}^{N}, \\
u_{n} \rightarrow u & \text { a.e. on } \mathbb{R}^{N} .
\end{aligned}
$$

Define

$$
\lambda_{\infty}=\lim _{R \rightarrow \infty} \varlimsup_{n \rightarrow \infty} \int_{|x| \geq R}\left|\Delta u_{n}\right|^{2} d x, \quad \nu_{\infty}=\lim _{R \rightarrow \infty} \varlimsup_{n \rightarrow \infty} \int_{|x| \geq R}\left|u_{n}\right|^{2 *} d x .
$$

Then it follows that

$$
\begin{aligned}
\|\nu\|^{2 / 2_{*}} & \leq S^{-1}\|\lambda\|, \\
\nu_{\infty}^{2 / 2_{*}} & \leq S^{-1} \lambda_{\infty}, \\
\lim _{n \rightarrow \infty}\left|\Delta u_{n}\right|_{2}^{2} & =|\Delta u|_{2}^{2}+\|\lambda\|+\lambda_{\infty}, \\
\varlimsup_{n \rightarrow \infty}\left|u_{n}\right|_{2_{*}^{*}}^{2_{*}} & =|u|_{2_{*}^{*}}^{2_{*}}+\|\nu\|+\nu_{\infty} .
\end{aligned}
$$

Moreover, if $u=0$ and $\|\nu\|^{2 / 2_{*}}=S^{-1}\|\lambda\|$, then $\lambda$ and $\nu$ are concentrated at a common single point.

Proof. See Appendix A. 
Lemma 3.2. Assume $0<\mu<\mu_{1}(\Omega)$. Any (PS)-sequence for $I$ is bounded.

Proof. It follows from standard arguments, since

$$
\|u\|_{\mu}=\left(\int_{\Omega}|\Delta u|^{2} d x-\mu \int_{\Omega}\left(u^{+}\right)^{2} d x\right)^{1 / 2}, \quad u \in E(\Omega)
$$

is a norm in $E(\Omega)$ and $2_{*}>2$.

Lemma 3.3. Assume $0<\mu<\mu_{1}(\Omega)$. Any sequence $\left(u_{n}\right) \subset E(\Omega)$ such that

$$
I\left(u_{n}\right) \rightarrow d<c^{*}:=\frac{2}{N} S^{N / 4} \quad \text { and } \quad I^{\prime}\left(u_{n}\right) \rightarrow 0
$$

contains a convergent subsequence.

Proof. By Lemma 3.2 it follows that, up to a subsequence,

$$
u_{n} \rightarrow u \quad \text { in } E(\Omega), \quad u_{n} \rightarrow u \quad \text { in } L^{2}(\Omega) \quad \text { and } \quad u_{n} \rightarrow u \quad \text { a.e. on } \Omega \text {. }
$$

For every $\varphi \in E(\Omega)$ we have

$$
\int_{\Omega} \Delta u_{n} \Delta \varphi d x-\mu \int_{\Omega}\left(u_{n}^{+}\right) \varphi d x=\int_{\Omega}\left(u_{n}^{+}\right)^{2_{*}-1} \varphi d x+o_{n}(1) .
$$

From the continuous embedding $E(\Omega) \hookrightarrow L^{2 *}(\Omega),\left(u_{n}^{+}\right)$is bounded in $L^{2 *}(\Omega)$ and consequently $\left(\left(u_{n}^{+}\right)^{2_{*}-1}\right)$ is bounded in $L^{2_{*} /\left(2_{*}-1\right)}(\Omega)$; we have also $u_{n}^{+} \rightarrow u^{+}$ almost everywhere on $\Omega$. Hence, as a consequence of the Brézis-Lieb lemma, see for instance [16, Lemma 4.8], $\left(u_{n}^{+}\right)^{2_{*}-1} \rightarrow\left(u^{+}\right)^{2_{*}-1}$ in $L^{2_{*} /\left(2_{*}-1\right)}(\Omega)$, and we obtain

$$
\int_{\Omega}\left(u_{n}^{+}\right)^{2_{*}-1} \varphi d x \rightarrow \int_{\Omega}\left(u^{+}\right)^{2_{*}-1} \varphi d x, \quad \text { for all } \varphi \in L^{2_{*}}(\Omega),
$$

in particular, (3.10) holds for any $\varphi \in E(\Omega)$. From $u_{n} \rightarrow u$ in $L^{2}(\Omega)$ we get

$$
\int_{\Omega}\left(u_{n}^{+}\right) \varphi d x \rightarrow \int_{\Omega}\left(u^{+}\right) \varphi d x, \quad \text { for all } \varphi \in E(\Omega) .
$$

Now, since $u_{n} \rightarrow u$ in $E(\Omega)$, we obtain

$$
\int_{\Omega} \Delta u_{n} \Delta \varphi d x=:\left\langle u_{n}, \varphi\right\rangle \rightarrow\langle u, \varphi\rangle:=\int_{\Omega} \Delta u \Delta \varphi d x, \quad \text { for all } \varphi \in E(\Omega) .
$$

Thus, taking $n \rightarrow \infty$ in (3.9) and using (3.10)-(3.12) we obtain

$$
\int_{\Omega} \Delta u \Delta \varphi d x-\mu \int_{\Omega}\left(u^{+}\right) \varphi d x=\int_{\Omega}\left(u^{+}\right)^{2_{*}-1} \varphi d x, \quad \text { for all } \varphi \in E(\Omega),
$$

that is, $u$ is a weak solution for the problem

$$
\begin{cases}\Delta^{2} u=\mu\left(u^{+}\right)+\left(u^{+}\right)^{2 *-1} & \text { in } \Omega \\ u, \Delta u=0 & \text { on } \partial \Omega\end{cases}
$$


and $u,-\Delta u$ are nonnegative in $\Omega$. Indeed, since $-\Delta: E(\Omega) \rightarrow L^{2}(\Omega)$ is an isomorphism [15], it follows, from (3.13), that

$$
\int_{\Omega} \Delta u(-w) d x=\mu \int_{\Omega}\left(u^{+}\right)\left[(-\Delta)^{-1} w\right] d x+\int_{\Omega}\left(u^{+}\right)^{2_{*}-1}\left[(-\Delta)^{-1} w\right] d x,
$$

for all $w \in L^{2}(\Omega)$, and, from the weak maximum principle,

$$
(-\Delta)^{-1} w \geq 0, \quad \text { for all } w \in L^{2}(\Omega) \text { and } w \geq 0,
$$

and thus

$$
\int_{\Omega} \Delta u(-w) d x \geq 0, \quad \text { if } w \geq 0 .
$$

Hence $u \in E(\Omega)$ and $-\Delta u \geq 0$ in $\Omega$. Consequently, by the weak maximum principle, $u \geq 0$ in $\Omega$.

With $\varphi=u$ in (3.13) we obtain

$$
|\Delta u|_{2}^{2}-\mu\left|u^{+}\right|_{2}^{2}=\left|u^{+}\right|_{2_{*}}^{2_{*}}
$$

and

$$
I(u)=\frac{1}{2}\left[|\Delta u|_{2}^{2}-\mu\left|u^{+}\right|_{2}^{2}\right]-\frac{1}{2_{*}}\left|u^{+}\right|_{2_{*}^{*}}^{2_{*}}=\left(\frac{1}{2}-\frac{1}{2_{*}}\right)\left|u^{+}\right|_{2_{*}^{*}}^{2_{*}} \geq 0 .
$$

Writing now $v_{n}=u_{n}-u$, see [28, p. 33] the Brézis-Lieb lemma leads to

$$
\left|u_{n}^{+}\right|_{2_{*}}^{2_{*}}=\left|u^{+}\right|_{2_{*}}^{2_{*}}+\left|v_{n}^{+}\right|_{2_{*}}^{2_{*}}+o_{n}(1) .
$$

From $u_{n} \rightarrow u$ in $L^{2}(\Omega)$, we also have

$$
\left|u_{n}^{+}\right|_{2}^{2}=\left|u^{+}\right|_{2}^{2}+\left|v_{n}^{+}\right|_{2}^{2}+o_{n}(1) .
$$

Using now (3.16) and (3.17) we have

$$
\begin{aligned}
I\left(u_{n}\right) & =\frac{1}{2}\left|\Delta u_{n}\right|_{2}^{2}-\frac{\mu}{2}\left|u_{n}^{+}\right|_{2}^{2}-\frac{1}{2_{*}}\left|u_{n}^{+}\right|_{2_{*}}^{2_{*}} \\
& =I(u)+\frac{1}{2}\left|\Delta v_{n}\right|_{2}^{2}-\frac{\mu}{2}\left|v_{n}^{+}\right|_{2}^{2}-\frac{1}{2_{*}}\left|v_{n}^{+}\right|_{2_{*}}^{2_{*}}+o_{n}(1),
\end{aligned}
$$

because $v_{n} \rightarrow 0$ in $E(\Omega)$. Assuming $I\left(u_{n}\right) \rightarrow d<c^{*}$, we obtain

$$
I(u)+\frac{1}{2}\left|\Delta v_{n}\right|_{2}^{2}-\frac{\mu}{2}\left|v_{n}^{+}\right|_{2}^{2}-\frac{1}{2_{*}}\left|v_{n}^{+}\right|_{2_{*}}^{2_{*}} \rightarrow d .
$$

Using again (3.16) and (3.17)

$$
\begin{aligned}
& I^{\prime}\left(u_{n}\right) u_{n}=\left|\Delta u_{n}\right|_{2}^{2}-\mu\left|u_{n}^{+}\right|_{2}^{2}-\left|u_{n}^{+}\right|_{2_{*}^{*}}^{2_{*}} \\
& \quad=\left|\Delta v_{n}\right|_{2}^{2}+2\left\langle v_{n}, u\right\rangle+|\Delta u|_{2}^{2}-\mu\left|u^{+}\right|_{2}^{2}-\mu\left|v_{n}^{+}\right|_{2}^{2}-\left|u^{+}\right|_{2_{*}^{*}}^{2_{*}}-\left|v_{n}^{+}\right|_{2_{*}}^{2_{*}}+o_{n}(1)
\end{aligned}
$$

and since $I^{\prime}\left(u_{n}\right) u_{n} \rightarrow 0$, we conclude, now using (3.14), that

$$
\left|\Delta v_{n}\right|_{2}^{2}-\mu\left|v_{n}^{+}\right|_{2}^{2}-\left|v_{n}^{+}\right|_{2_{*}}^{2_{*}} \rightarrow|\Delta u|_{2}^{2}-\mu\left|u^{+}\right|_{2}^{2}-\left|u^{+}\right|_{2_{*}}^{2_{*}}=0 .
$$

So, we may assume that $\left|\Delta v_{n}\right|_{2}^{2}-\mu\left|v_{n}^{+}\right|_{2}^{2} \rightarrow b$ and $\left|v_{n}^{+}\right|_{2_{*}^{*}}^{2_{*}} \rightarrow b$. 
Since $v_{n} \rightarrow 0$ in $L^{2}(\Omega)$, in particular, $v_{n}^{+} \rightarrow 0$ in $L^{2}(\Omega)$. Then it follows that $\left|\Delta v_{n}\right|_{2}^{2} \rightarrow b$. By the definition of $S$ we have,

$$
\left|\Delta v_{n}\right|_{2}^{2} \geq S\left|v_{n}\right|_{2_{*}}^{2} \geq S\left|v_{n}^{+}\right|_{2_{*}}^{2}
$$

which implies $b \geq S b^{2 / 2_{*}}=S b^{(N-4) / N}$. Thus, either $b=0$ or $b \geq S^{N / 4}$.

From (3.18),

$$
I(u)+\left(\frac{1}{2}-\frac{1}{2_{*}}\right) b=I(u)+\frac{2}{N} b=d
$$

and from (3.15), $d \geq 2 / N b$. If $b \geq S^{N / 4}$ we obtain

$$
c^{*}=\frac{2}{N} S^{N / 4} \leq \frac{2}{N} b \leq d<c^{*},
$$

a contradiction. Hence, $b=0$, and the proof is complete, because

$$
\left\|u_{n}-u\right\|^{2}=\left\|v_{n}\right\|^{2}=\left|\Delta v_{n}\right|_{2}^{2} \rightarrow 0, \quad \text { that is, } u_{n} \rightarrow u \text { in } E(\Omega) .
$$

Lemma 3.4. Assume $0<\mu<\mu_{1}(\Omega)$. Any sequence $\left(u_{n}\right) \subset V$ such that

$$
I_{\mu}\left(u_{n}\right) \rightarrow c<S, \quad\left\|I_{\mu}^{\prime}\left(u_{n}\right)\right\|_{*} \rightarrow 0,
$$

contains a convergent subsequence, where $\|\cdot\|_{*}$ denotes the norm of the derivative of $\left.I_{\mu}\right|_{V}$, and is given by

$$
\left\|I_{\mu}^{\prime}(u)\right\|_{*}=\min _{\lambda \in \mathbb{R}}\left\|I_{\mu}^{\prime}(u)-\lambda \psi^{\prime}(u)\right\|, \quad \text { for all } u \in V .
$$

Proof. If $\left(u_{n}\right)$ satisfies (3.19), then $0 \leq I_{\mu}\left(u_{n}\right) \rightarrow c$ and

$$
\left\|I_{\mu}^{\prime}\left(u_{n}\right)\right\|_{*}=\left\|I_{\mu}^{\prime}\left(u_{n}\right)-\bar{\lambda}_{n} \psi^{\prime}\left(u_{n}\right)\right\| \rightarrow 0, \quad \text { for } \bar{\lambda}_{n} \in \mathbb{R} .
$$

So, there exists $\left(\sigma_{n}\right) \subset[0,+\infty), \sigma_{n} \rightarrow 0$ such that

$$
\left|\int_{\Omega} \Delta u_{n} \Delta w d x-\mu \int_{\Omega}\left(u_{n}^{+}\right) w d x-\lambda_{n} \int_{\Omega}\left(u_{n}^{+}\right)^{2_{*}-1} w d x\right| \leq \sigma_{n}\|w\|,
$$

for all $w \in E(\Omega), \lambda_{n} \in \mathbb{R}$. The sequence $\left(u_{n}\right)$ is bounded in $E(\Omega)$. Indeed,

$$
\left\|u_{n}\right\|^{2}=\left\|u_{n}\right\|^{2}-\mu\left|u_{n}^{+}\right|_{2}^{2}+\mu\left|u_{n}^{+}\right|_{2}^{2}=c+o_{n}(1)+\mu\left|u_{n}^{+}\right|_{2}^{2},
$$

and from the continuous embedding of $L^{2_{*}}(\Omega)$ into $L^{2}(\Omega)$, it follows that $\left(u_{n}\right)$ is bounded in $E(\Omega)$. Thus

$$
\left|\int_{\Omega}\left[\left|\Delta u_{n}\right|^{2}-\mu\left(u_{n}^{+}\right)^{2}\right] d x-\lambda_{n} \int_{\Omega}\left(u_{n}^{+}\right)^{2 *} d x\right| \leq \sigma_{n}\left\|u_{n}\right\| \Rightarrow I_{\mu}\left(u_{n}\right)-\lambda_{n} \rightarrow 0,
$$

that is, $\lambda_{n} \rightarrow c \geq 0$.

If $c=0$, then

$$
\begin{aligned}
0 & \leq\left(1-\frac{\mu}{\mu_{1}(\Omega)}\right)\left\|u_{n}\right\|^{2}=\left\|u_{n}\right\|^{2}-\frac{\mu}{\mu_{1}(\Omega)}\left\|u_{n}\right\|^{2} \\
& \leq\left\|u_{n}\right\|^{2}-\mu\left|u_{n}\right|^{2} \leq\left\|u_{n}\right\|^{2}-\mu\left|u_{n}^{+}\right|^{2}=I_{\mu}\left(u_{n}\right) \rightarrow 0,
\end{aligned}
$$

and $\left(u_{n}\right)$ converges strongly to 0 in $E(\Omega)$. 
If $c>0$ then $\lambda_{n}>0$ for $n$ big enough. So, put $v_{n}=\lambda_{n}^{1 /\left(2_{*}-2\right)} u_{n}$. Taking $I$ given by (2.6),

$$
\begin{aligned}
I\left(v_{n}\right)= & \frac{1}{2} \int_{\Omega}\left[\left|\Delta\left(\lambda_{n}^{1 /\left(2_{*}-2\right)} u_{n}\right)\right|^{2}-\mu\left(\lambda_{n}^{1 /\left(2_{*}-2\right)} u_{n}^{+}\right)^{2}\right] d x \\
& -\frac{1}{2_{*}} \int_{\Omega}\left(\lambda_{n}^{1 /\left(2_{*}-2\right)} u_{n}^{+}\right)^{2_{*}} d x \\
= & \frac{1}{2} \lambda_{n}^{2 /\left(2_{*}-2\right)} I_{\mu}\left(u_{n}\right)-\frac{1}{2_{*}} \lambda_{n}^{2_{*} /\left(2_{*}-2\right)} \\
& \rightarrow \frac{1}{2} c^{2 /\left(2_{*}-2\right)} c-\frac{1}{2_{*}} c^{2_{*} /\left(2_{*}-2\right)}=\frac{2}{N} c^{N / 4}
\end{aligned}
$$

and

$$
\begin{aligned}
\left|I^{\prime}\left(v_{n}\right) w\right|= & \mid \int_{\Omega}\left[\Delta\left(\lambda_{n}^{1 /\left(2_{*}-2\right)} u_{n}\right) \Delta w-\mu\left(\lambda_{n}^{1 /\left(2_{*}-2\right)} u_{n}^{+}\right) w\right] d x \\
& -\int_{\Omega}\left(\lambda_{n}^{1 /\left(2_{*}-2\right)} u_{n}^{+}\right)^{2_{*}-1} w d x \mid \\
= & \lambda_{n}^{1 /\left(2_{*}-2\right)}\left|\int_{\Omega}\left[\Delta u_{n} \Delta w-\mu\left(u_{n}^{+}\right) w-\lambda_{n}\left(u_{n}^{+}\right)^{2_{*}-1} w\right] d x\right| \\
\leq & \lambda_{n}^{1 /\left(2_{*}-2\right)} \sigma_{n}\|w\|,
\end{aligned}
$$

for all $w \in E(\Omega)$. Hence

$$
I\left(v_{n}\right) \rightarrow \frac{2}{N} c^{N / 4}<\frac{2}{N} S^{N / 4}=c^{*} \quad \text { and } \quad I^{\prime}\left(v_{n}\right) \rightarrow 0 .
$$

From Lemma $3.3,\left(v_{n}\right)$ contains a convergent subsequence, and then $\left(u_{n}\right)$ also contains a convergent subsequence.

\section{Multiplicity of solutions}

We first recall a classical result in the theory of the Lusternik-Schnirelman category [19].

Theorem 4.1 ([28, Theorem 5.20]). Let $X$ be a Banach space, $\varphi \in \mathcal{C}^{1}(X, \mathbb{R})$, $\psi \in \mathcal{C}^{2}(X, \mathbb{R}), V=\{v \in X: \psi(v)=1\}$ and for all $v \in V, \psi^{\prime}(v) \neq 0$. If $\left.\varphi\right|_{V}$ is bounded from below and satisfies the (PS) $)_{c}$-condition for any $c \in\left[\inf _{V} \varphi, d\right]$, then $\left.\varphi\right|_{V}$ has a minimum and the set $\varphi^{d}:=\{v \in V: \varphi(v) \leq d\}$ contains at least $\operatorname{cat}_{\varphi^{d}}\left(\varphi^{d}\right)$ critical points of $\left.\varphi\right|_{V}$.

In our context, $X=E(\Omega), \psi(u)=\int_{\Omega}\left(u^{+}\right)^{2_{*}} d x$ and $\varphi=I_{\mu}$.

Lemma 4.2. Let $N \geq 8$ and $0<\mu<\mu_{1}(\Omega)$. There exists $v \in E(\Omega) \backslash\{0\}$, with $v>0$ in $\Omega$ such that

$$
\frac{\|v\|_{\mu}^{2}}{|v|_{2_{*}}^{2}}=\frac{|\Delta v|_{2}^{2}-\mu|v|_{2}^{2}}{|v|_{2_{*}}^{2}}<S .
$$

Proof. See Appendix B. 
Lemma 4.3. If $0<\mu<\mu_{1}(\Omega)$ and $N \geq 8$, then $m(\mu, \Omega)<S$ and there exists $u \in V$, such that $u,-\Delta u>0$ in $\Omega$ and $I_{\mu}(u)=m(\mu, \Omega)$, with $m(\mu, \Omega)$ as defined by (2.9).

Proof. By Lemma 4.2, there exists $v \in E(\Omega) \backslash\{0\}$ nonnegative such that

$$
\frac{|\Delta v|_{2}^{2}-\mu|v|_{2}^{2}}{|v|_{2_{*}}^{2}}<S
$$

Setting $w=v /|v|_{2_{*}}$, we have $w \in V$ and

$$
I_{\mu}(w)=|\Delta w|_{2}^{2}-\mu\left|w^{+}\right|_{2}^{2}=|\Delta w|_{2}^{2}-\mu|w|_{2}^{2}=\frac{|\Delta v|_{2}^{2}-\mu|v|_{2}^{2}}{|v|_{2_{*}}^{2}}<S,
$$

and therefore

$$
m(\mu, \Omega)=\inf _{u \in V} I_{\mu}(u) \leq I_{\mu}(w)<S .
$$

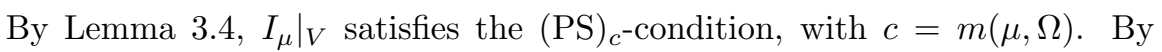
Theorem 4.1, $\left.I_{\mu}\right|_{V}$ has a minimum, that is, there exists $u \in V$ such that

$$
I_{\mu}(u)=m(\mu, \Omega)=\min _{u \in V} I_{\mu}(u) .
$$

Now we show that $u,-\Delta u>0$ in $\Omega$. Since $u$ is such that

$$
\left|u^{+}\right|_{2_{*}}^{2_{*}}=1, \quad I_{\mu}(u)=|\Delta u|_{2}^{2}-\mu\left|u^{+}\right|_{2}^{2}=m(\mu, \Omega)>0,
$$

it follows from Lagrange multipliers theorem that $u$ satisfies

$$
\int_{\Omega} \Delta u \Delta v d x=\mu \int_{\Omega}\left(u^{+}\right) v d x+m(\mu, \Omega) \int_{\Omega}\left(u^{+}\right)^{2_{*}-1} v d x, \quad \text { for all } v \in E(\Omega) .
$$

So,

$$
\int_{\Omega} \Delta u(-w) d x=\mu \int_{\Omega}\left(u^{+}\right)\left[(-\Delta)^{-1} w\right] d x+m(\mu, \Omega) \int_{\Omega}\left(u^{+}\right)^{2_{*}-1}\left[(-\Delta)^{-1} w\right] d x,
$$

for all $w \in L^{2}(\Omega)$ and $(-\Delta)^{-1} w \geq 0$, for all $w \in L^{2}(\Omega), w \geq 0$. Thus,

$$
\int_{\Omega} \Delta u(-w) d x \geq 0, \quad \text { for all } w \geq 0,
$$

and therefore $-\Delta u \geq 0$ and consequently $u \geq 0$. Since $u$ is nontrivial, it follows by the strong maximum principle that $u,-\Delta u>0$ in $\Omega$.

Lemma 4.4. If $\Omega_{1}$ and $\Omega_{2}$ are regular bounded domains in $\mathbb{R}^{N}, N \geq 8$, such that $\Omega_{1} \subset \subset \Omega_{2}$ and $0<\mu<\mu_{1}\left(\Omega_{2}\right)$, then $m\left(\mu, \Omega_{1}\right)>m\left(\mu, \Omega_{2}\right)$.

Proof. First we recall that $\Omega_{1} \subset \subset \Omega_{2}$ implies that $\mu_{1}\left(\Omega_{2}\right)<\mu_{1}\left(\Omega_{1}\right)$. So, let $u \in E\left(\Omega_{1}\right)$ be a function such that $u,-\Delta u>0$ in $\Omega_{1}$ and

$$
\int_{\Omega_{1}}\left(u^{+}\right)^{2_{*}} d x=1, \quad \int_{\Omega_{1}}\left[|\Delta u|^{2}-\mu\left(u^{+}\right)^{2}\right] d x=m\left(\mu, \Omega_{1}\right),
$$


and take $w$ as the solution for

$$
\begin{cases}-\Delta w=\widetilde{-\Delta u} & \text { in } \Omega_{2}, \\ w=0 & \text { on } \partial \Omega_{2},\end{cases}
$$

where $\sim$ denotes the zero extension outside $\Omega_{1}$. Note that $w \geq 0$ in $\Omega_{2}$ and $w>u$ in $\Omega_{1}$. Set $\bar{w}=w /|w|_{2_{*}, \Omega_{2}}$. Then $\left|\bar{w}^{+}\right|_{2_{*}, \Omega_{2}}=1$ and

$$
\begin{aligned}
m\left(\mu, \Omega_{2}\right) & \leq \int_{\Omega_{2}}\left[|\Delta \bar{w}|^{2}-\mu\left(\bar{w}^{+}\right)^{2}\right] d x=\frac{1}{|w|_{2_{*}, \Omega_{2}}^{2}} \int_{\Omega_{2}}\left[|\Delta w|^{2}-\mu\left(w^{+}\right)^{2}\right] d x \\
& <\int_{\Omega_{2}}\left[|\Delta w|^{2}-\mu\left(w^{+}\right)^{2}\right] d x<\int_{\Omega_{1}}\left[|\Delta u|^{2}-\mu\left(u^{+}\right)^{2}\right] d x=m\left(\mu, \Omega_{1}\right) .
\end{aligned}
$$

LEMmA 4.5. If $\Omega=B_{\rho}(0) \subset \mathbb{R}^{N}, N \geq 8$ and $0<\mu<\mu_{1}(\Omega)$, then $m(\mu, \rho)$ is attained by a function $u$ such that $u,-\Delta u>0$ in $B_{\rho}(0)$ and $u,-\Delta u$ are radially symmetric. Moreover, such a solution $u$ is unique.

ProOF. Let $u$ be a function such that $u,-\Delta u>0$ in $B_{\rho}(0)$ and that realizes $m(\mu, \rho)$. Denote by $u^{*}$ and $(-\Delta u)^{*}$ the Schwarz symmetrization of $u$ and $-\Delta u$, respectively. If $v$ is the solution of

$$
\begin{cases}-\Delta v=(-\Delta u)^{*} & \text { in } B_{\rho}(0) \\ v=0 & \text { on } \partial B_{\rho}(0)\end{cases}
$$

then $v=v^{*}$. We just need to prove that $u=v$. By [3], see also [6, Lemma 2.8], we have $v \geq u^{*}$ and

$$
\left|v>u^{*}\right|=0 \Leftrightarrow-\Delta u=(-\Delta u)^{*} .
$$

If $\left|v>u^{*}\right|>0$, set $w=v /|v|_{2_{*}}$. So $\left|w^{+}\right|_{2_{*}}=1$ and

$$
\begin{aligned}
m(\mu, \rho) & \leq \int_{B_{\rho}(0)}\left[|\Delta w|^{2}-\mu\left(w^{+}\right)^{2}\right] d x=\frac{1}{|v|_{2_{*}}^{2}} \int_{B_{\rho}(0)}\left[|\Delta v|^{2}-\mu\left(v^{+}\right)^{2}\right] d x \\
& <\frac{1}{|v|_{2_{*}}^{2}} \int_{B_{\rho}(0)}\left[\left|(-\Delta u)^{*}\right|^{2}-\mu\left(u^{*}\right)^{2}\right] d x \\
& <\frac{1}{\left|u^{*}\right|_{2_{*}}^{2}} \int_{B_{\rho}(0)}\left[\left|(-\Delta u)^{*}\right|^{2}-\mu\left(u^{*}\right)^{2}\right] d x \\
& =\frac{1}{\left|u^{+}\right|_{2_{*}}^{2}} \int_{B_{\rho}(0)}\left[|-\Delta u|^{2}-\mu\left(u^{+}\right)^{2}\right] d x=m(\mu, \rho),
\end{aligned}
$$

which is a contradiction. Thus, $-\Delta u=(-\Delta u)^{*}$ and since $u$ and $v$ are solutions for the problem

it follows that $u=v$.

$$
\begin{cases}-\Delta w=(-\Delta u)^{*} & \text { in } B_{\rho}(0) \\ w=0 & \text { on } \partial B_{\rho}(0)\end{cases}
$$

Finally we mention that the uniqueness of $u$ can be proved arguing as in $[12$, Section 3] by means of comparison principle for radial function [20]. 
Now define $\beta: V \rightarrow \mathbb{R}^{N}$ by

$$
\beta(u)=\frac{\int_{\Omega}|\Delta u|^{2} x d x}{\int_{\Omega}|\Delta u|^{2} d x} .
$$

Lemma 4.6. If $\left(u_{n}\right) \subset V$ is such that $\left\|u_{n}\right\|^{2}=\left|\Delta u_{n}\right|_{2}^{2} \rightarrow S$, then

$$
\operatorname{dist}\left(\beta\left(u_{n}\right), \Omega\right) \rightarrow 0 \text {. }
$$

Proof. Suppose, by contradiction, that $\operatorname{dist}\left(\beta\left(u_{n}\right), \Omega\right) \not \rightarrow 0$. So, there exists $r>0$ such that, up to a subsequence, $\operatorname{dist}\left(\beta\left(u_{n}\right), \Omega\right)>r$.

Set $v_{n}=u_{n} /\left|\Delta u_{n}\right|_{2} \in E(\Omega)$ and $w_{n}$ as the Newtonian potential of $\left|\widetilde{-\Delta v_{n}}\right| \in$ $L^{2}\left(\mathbb{R}^{N}\right)$, where $\sim$ denotes the zero extension outside $\Omega$. Then, by [15, Theorem 9.9], we know that $w_{n} \in \mathcal{D}^{2,2}\left(\mathbb{R}^{N}\right)$ and

$$
-\Delta w_{n}=\left|\widetilde{-\Delta v_{n}}\right| \text { a.e. in } \mathbb{R}^{N} \text {. }
$$

In particular, $\left(w_{n}\right)$ is a bounded sequence in $\mathcal{D}^{2,2}\left(\mathbb{R}^{N}\right)$. Then, up to a subsequence,

$$
\begin{aligned}
w_{n} \rightarrow w & \text { in } \mathcal{D}^{2,2}\left(\mathbb{R}^{N}\right), \\
\left|\Delta\left(w_{n}-w\right)\right|^{2} \stackrel{*}{\rightarrow} \lambda & \text { in the sense of measures on } \mathbb{R}^{N}, \\
\left|w_{n}-w\right|^{2 *} \stackrel{*}{\rightarrow} \nu & \text { in the sense of measures on } \mathbb{R}^{N}, \\
w_{n} \rightarrow w & \text { a.e. on } \mathbb{R}^{N} .
\end{aligned}
$$

We have by Lemma 3.1 , taking into account that $\lambda_{\infty}=0$ and $w_{n} \geq\left|\widetilde{v_{n}}\right|$ in $\mathbb{R}^{N}$,

$$
\begin{aligned}
1 & =|\Delta w|_{2}^{2}+\|\lambda\|, \\
\frac{1}{S^{2_{*} / 2}} & \leq|w|_{2_{*}^{*}}^{2_{*}}+\|\nu\|,
\end{aligned}
$$

and

$$
\|\nu\|^{2 / 2_{*}} \leq \frac{1}{S}\|\lambda\|, \quad|w|_{2_{*}}^{2} \leq \frac{1}{S}|\Delta w|_{2}^{2} .
$$

It follows that the pair $\left(|\Delta w|_{2}^{2},\|\lambda\|\right) \in\{(1,0),(0,1)\}$. Indeed, from (4.6)

$$
\|\nu\| \leq \frac{1}{S^{2_{*} / 2}}\|\lambda\|^{2_{*} / 2}, \quad|w|_{2_{*}^{*}}^{2_{*}}=\left(|w|_{2_{*}}^{2_{*}}\right)^{2_{*} / 2} \leq \frac{1}{S^{2_{*} / 2}}|\Delta w|_{2}^{2_{*}},
$$

and so

that is

$$
\frac{1}{S^{2_{*} / 2}} \leq|w|_{2_{*}^{*}}^{2_{*}}+\|\nu\| \leq \frac{1}{S^{2_{*} / 2}}\left[|\Delta w|_{2}^{2_{*}}+\|\lambda\|^{2_{*} / 2}\right],
$$

$$
|\Delta w|_{2}^{2_{*}}+\|\lambda\|^{2_{*} / 2} \geq 1
$$

From (4.4), (4.7) and since $2_{*} / 2>1$, we get that the pair $\left(|\Delta w|_{2}^{2},\|\lambda\|\right) \in$ $\{(1,0),(0,1)\}$. 
Suppose now that $|\Delta w|_{2}^{2}=1$ and $\|\lambda\|=0$. So, by (4.6), $\|\nu\|=0$ which implies, by (4.5),

$$
\frac{1}{S} \leq|w|_{2 *}^{2} \leq \frac{1}{S}|\Delta w|_{2}^{2}=\frac{1}{S}
$$

and so $|\Delta w|_{2}^{2} /|w|_{2_{*}}^{2}=S$. Then, up to a multiple, $w$ is a non-negative non-trivial solution of the equation

$$
\Delta^{2} w=w^{2_{*}-1} \quad \text { in } \mathbb{R}^{N}
$$

and therefore, $w,-\Delta w>0$ in $\mathbb{R}^{N}$. But, for all $\varphi \in \mathcal{C}_{c}^{\infty}\left(\mathbb{R}^{N}\right)$ we have

$$
\int_{\mathbb{R}^{N}}\left|\Delta w_{n}-\Delta w\right|^{2} \varphi d x \rightarrow \int_{\mathbb{R}^{N}} \varphi d \lambda=0
$$

which implies, in particular,

$$
\int_{\Omega}\left|\Delta w_{n}-\Delta w\right|^{2} \varphi d x+\int_{\mathbb{R}^{N} \backslash \Omega}|\Delta w|^{2} \varphi d x \rightarrow 0, \quad \text { for all } \varphi \in \mathcal{C}_{c}^{\infty}\left(\mathbb{R}^{N} \backslash \Omega\right),
$$

and then $-\Delta w=0$ in $\mathbb{R}^{N} \backslash \Omega$, which leads a contradiction.

Thus, $|\Delta w|_{2}^{2}=0$ (and from (4.6), it follows that $w=0$ ) and $\|\lambda\|=1$. From (4.5) and (4.6), we get $\|\nu\|^{2 / 2_{*}}=S^{-1}\|\lambda\|$. Therefore, by Lemma 3.1, it follows that $\lambda$ concentrates at a single point $y \in \mathbb{R}^{N}$.

We infer that $y \in \bar{\Omega}$. Indeed, by contradiction suppose $y \in \mathbb{R}^{N} \backslash \bar{\Omega}$. Take $\psi \in \mathcal{C}_{c}^{\infty}\left(\mathbb{R}^{N}\right)$ such that $\psi \equiv 1$ in $B_{R}(y)$, for some $R>0$, and $\operatorname{supp}(\psi) \cap \bar{\Omega}=\emptyset$. So,

$$
1=\lambda(\{y\})=\int_{\mathbb{R}^{N}} \psi d \lambda=\lim _{n \rightarrow \infty} \int_{\mathbb{R}^{N}} \psi\left|\Delta w_{n}\right|^{2} d x=0,
$$

which is clearly a contradiction. Hence, $y \in \bar{\Omega}$ and taking $\eta \in \mathcal{C}_{c}^{\infty}\left(\mathbb{R}^{N}\right), \eta \equiv 1$ in $\bar{\Omega}$, we have

$$
\begin{aligned}
\beta\left(u_{n}\right) & =\frac{\int_{\Omega}\left|\Delta u_{n}\right|^{2} x d x}{\int_{\Omega}\left|\Delta u_{n}\right|^{2} d x}=\int_{\Omega}\left|\Delta v_{n}\right|^{2} x d x \\
& =\int_{\mathbb{R}^{N}}\left|\Delta w_{n}\right|^{2} x \eta(x) d x \rightarrow \int_{\mathbb{R}^{N}} x \eta(x) d \lambda=y \eta(y)=y \in \bar{\Omega},
\end{aligned}
$$

which contradicts our initial hypothesis.

Without loss of generality we can assume that $0 \in \Omega$. Let $r>0$ be small enough such that

$$
\Omega_{r}^{+}:=\left\{u \in \mathbb{R}^{N}: \operatorname{dist}(u, \bar{\Omega}) \leq r\right\} \quad \text { and } \quad \Omega_{r}^{-}:=\{u \in \Omega: \operatorname{dist}(u, \partial \Omega) \geq r\}
$$

are homotopically equivalent to $\Omega$ and such that $B_{r}(0) \subset \subset \Omega$. We also set

$$
I_{\mu}^{m(\mu, r)}:=\left\{u \in V: I_{\mu}(u) \leq m(\mu, r)\right\},
$$

which is nonempty; see Lemma 4.4 . 
Lemma 4.7. There exists $0<\bar{\mu}<\mu_{1}(\Omega)$ such that, for $0<\mu<\bar{\mu}$,

$$
u \in I_{\mu}^{m(\mu, r)} \Rightarrow \beta(u) \in \Omega_{r}^{+} .
$$

Proof. If $u \in V$, then by the Hölder inequality,

$$
\left|u^{+}\right|_{2}^{2} \leq\left|u^{+}\right|_{2_{*}}^{2}|\Omega|^{\left(2_{*}-2\right) / 2_{*}}=|\Omega|^{4 / N} .
$$

By Lemma 4.6, there exists $\varepsilon>0$ such that

$$
u \in V, \quad\|u\|^{2} \leq S+\varepsilon \Rightarrow \beta(u) \in \Omega_{r}^{+} .
$$

Set $\bar{\mu}:=\varepsilon /|\Omega|^{4 / N}$, for $\varepsilon>0$ sufficiently small such that $0<\bar{\mu}<\mu_{1}(\Omega)$. Hence, if $0<\mu<\bar{\mu}$ and $u \in I_{\mu}^{m(\mu, r)}$, we obtain, from (4.8) and Lemma 4.3,

$$
\begin{aligned}
\|u\|^{2} & =\|u\|^{2}-\mu\left|u^{+}\right|_{2}^{2}+\mu\left|u^{+}\right|_{2}^{2}=I_{\mu}(u)+\mu\left|u^{+}\right|_{2}^{2} \\
& \leq m(\mu, r)+\bar{\mu}\left|u^{+}\right|_{2}^{2}<S+\frac{\varepsilon}{|\Omega|^{4 / N}}|\Omega|^{4 / N}=S+\varepsilon,
\end{aligned}
$$

so that $\beta(u) \in \Omega_{r}^{+}$.

Let $\bar{\mu}$ as in Lemma 4.7. For each $0<\mu<\bar{\mu}$ we define $\gamma_{\mu}: \Omega_{r}^{-} \rightarrow I_{\mu}^{m(\mu, r)}$ by

$$
\gamma_{\mu}(y): \Omega \rightarrow \mathbb{R}, \quad x \mapsto \gamma_{\mu}(y)(x)=\frac{w_{y}(x)}{\left|w_{y}\right|_{2_{*}}},
$$

where $w_{y}$ is the solution for the problem

$$
\left\{\begin{array}{ll}
-\Delta w_{y}=z_{y} & \text { in } \Omega, \\
w_{y}=0 & \text { on } \partial \Omega,
\end{array} \quad \text { with } \quad z_{y}(x)= \begin{cases}-\Delta v_{\mu}(x-y) & \text { if } x \in B_{r}(y), \\
0 & \text { if } x \in \Omega \backslash B_{r}(y),\end{cases}\right.
$$

where, see Lemma $4.5, v_{\mu}$ is radially symmetric with respect to zero, $v_{\mu},-\Delta v_{\mu}>$ 0 in $B_{r}(0)$ and

$$
\int_{B_{r}(0)}\left(v_{\mu}^{+}\right)^{2 *} d x=1, \quad \int_{B_{r}(0)}\left[\left|\Delta v_{\mu}\right|^{2}-\mu\left(v_{\mu}^{+}\right)^{2}\right] d x=m(\mu, r) .
$$

REMARK 4.8. Arguing as in the proof of Lemma 2.2, we get that $v_{\mu} \in$ $C^{4}\left(\overline{B_{r}(0)}\right)$.

Lemma 4.9. Let $0<\mu<\bar{\mu}$, where $\bar{\mu}$ is given in Lemma 4.7. Then $\gamma_{\mu}: \Omega_{r}^{-} \rightarrow$ $I_{\mu}^{m(\mu, r)}$ is well defined, continuous and

$$
\left(\beta \circ \gamma_{\mu}\right)(y)=y, \quad \text { for all } y \in \Omega_{r}^{-} .
$$

Proof. First observe that [15, Theorem 9.15] guarantees that $\gamma_{\mu}(y) \in E(\Omega)$ and, by the strong maximum principle, we have $w_{y}(x)>v_{\mu}(x-y)$, for all $x \in B_{r}(y)$ and $y \in \Omega_{r}^{-}$. Then

$$
\begin{gathered}
\int_{\Omega}\left|\Delta w_{y}\right|^{2} d x=\int_{B_{r}(0)}\left|\Delta v_{\mu}\right|^{2} d x, \\
\int_{\Omega}\left|w_{y}\right|^{2} d x>\int_{B_{r}(0)}\left|v_{\mu}\right|^{2} d x,
\end{gathered}
$$




$$
\int_{\Omega}\left|w_{y}\right|^{2_{*}} d x>\int_{B_{r}(0)}\left|v_{\mu}\right|^{2_{*}} d x=1
$$

So,

$$
\begin{aligned}
I_{\mu}\left(\gamma_{\mu}(y)\right) & =I_{\mu}\left(\frac{w_{y}(x)}{\left|w_{y}\right|_{2_{*}}}\right)=\frac{1}{\left|w_{y}\right|_{2_{*}}^{2}}\left[\int_{\Omega}\left|\Delta w_{y}\right|^{2} d x-\mu \int_{\Omega}\left(w_{y}^{+}\right)^{2} d x\right] \\
& <\int_{\Omega}\left|\Delta w_{y}\right|^{2} d x-\mu \int_{\Omega}\left(w_{y}^{+}\right)^{2} d x \\
& \leq \int_{B_{r}(0)}\left|\Delta v_{\mu}\right|^{2} d x-\mu \int_{B_{r}(0)}\left(v_{\mu}^{+}\right)^{2} d x=m(\mu, r),
\end{aligned}
$$

that is, $\gamma_{\mu}(y) \in I_{\mu}^{m(\mu, r)}$ for every $y \in \Omega_{r}^{-}$and so $\gamma_{\mu}: \Omega_{r}^{-} \rightarrow I_{\mu}^{m(\mu, r)}$ is well defined.

The continuity of $\gamma_{\mu}$ is a consequence of the regularity of $v_{\mu}$. To prove that $\gamma_{\mu}$ is continuous, it is enough to prove that $\bar{\gamma}_{\mu}: \Omega_{r}^{-} \rightarrow E(\Omega)$, defined by $\bar{\gamma}_{\mu}(y)(x)=w_{y}(x)$, is continuous. If $y_{n} \rightarrow y$ in $\Omega_{r}^{-}$, then

$$
\begin{aligned}
\left\|\bar{\gamma}_{\mu}\left(y_{n}\right)-\bar{\gamma}_{\mu}(y)\right\|^{2} & =\left|\Delta\left(\bar{\gamma}_{\mu}\left(y_{n}\right)-\bar{\gamma}_{\mu}(y)\right)\right|_{2}^{2} \\
& =\left|\Delta w_{y_{n}}-\Delta w_{y}\right|_{2}^{2}=\left|z_{y_{n}}-z_{y}\right|_{2}^{2} \\
& =\left|z_{y_{n}}\right|_{2}^{2}-2 \int_{\Omega} z_{y_{n}}(x) z_{y}(x) d x+\left|z_{y}\right|_{2}^{2} \\
& =2\left[\int_{B_{r}(0)}\left|\Delta v_{\mu}(z)\right|^{2} d z-\int_{\Omega} z_{y_{n}}(x) z_{y}(x) d x\right] \rightarrow 0
\end{aligned}
$$

because $\Delta v_{\mu}: \overline{B_{r}(0)} \rightarrow \mathbb{R}$ is continuous. Finally, for every $y \in \Omega_{r}^{-}$,

$$
\begin{aligned}
\left(\beta \circ \gamma_{\mu}\right)(y) & =\frac{\int_{\Omega}\left|\Delta\left(\frac{w_{y}}{\left|w_{y}\right|_{2_{*}}}\right)\right|^{2} x d x}{\int_{\Omega}\left|\Delta\left(\frac{w_{y}}{\left|w_{y}\right|_{2_{*}}}\right)\right|^{2} d x}=\frac{\int_{\Omega}\left|\Delta w_{y}\right|^{2} x d x}{\int_{\Omega}\left|\Delta w_{y}\right|^{2} d x} \\
& =\frac{\int_{B_{r}(y)}\left|\Delta v_{\mu}(x-y)\right|^{2} x d x}{\int_{B_{r}(y)}\left|\Delta v_{\mu}(x-y)\right|^{2} d x}=\frac{\int_{B_{r}(0)}\left|\Delta v_{\mu}(z)\right|^{2}(z+y) d z}{\int_{B_{r}(0)}\left|\Delta v_{\mu}(z)\right|^{2} d z} \\
& =\frac{\int_{B_{r}(0)}\left|\Delta v_{\mu}(z)\right|^{2} z d z}{\int_{B_{r}(0)}\left|\Delta v_{\mu}(z)\right|^{2} d z}+\frac{y \int_{B_{r}(0)}\left|\Delta v_{\mu}(z)\right|^{2} d z}{\int_{B_{r}(0)}\left|\Delta v_{\mu}(z)\right|^{2} d z}=y
\end{aligned}
$$

because $\Delta v_{\mu}$ is radially symmetric.

Lemma 4.10. If $N \geq 8$ and $0<\mu<\bar{\mu}$, where $\bar{\mu}$ is given in Lemma 4.7 , then

$$
\operatorname{cat}_{I_{\mu}^{m(\mu, r)}}\left(I_{\mu}^{m(\mu, r)}\right) \geq \operatorname{cat}_{\Omega}(\Omega) .
$$


Proof. If $\operatorname{cat}_{\left.I_{\mu(\mu, r)}^{m(}\right)}\left(I_{\mu}^{m(\mu, r)}\right)=\infty$, then there is nothing to do.

If cat $_{I_{\mu}^{m(\mu, r)}}\left(I_{\mu}^{m(\mu, r)}\right)=n$, then $I_{\mu}^{m(\mu, r)}=A_{1} \cup \ldots \cup A_{n}$, where $A_{j}$ is closed and contractible in $I_{\mu}^{m(\mu, r)}$, for all $j=1, \ldots, n$.

For each $j=1, \ldots, n$, let $h_{j}:[0,1] \times A_{j} \rightarrow I_{\mu}^{m(\mu, r)}$ be a continuous map and $w_{j} \in I_{\mu}^{m(\mu, r)}$ such that

$$
h_{j}(0, u)=u, \quad h_{j}(1, u)=w_{j}, \quad \text { for all } u \in A_{j} .
$$

Consider $B_{j}=\gamma_{\mu}^{-1}\left(A_{j}\right)$, where $\gamma_{\mu}$ is given by (4.9). The sets $B_{j}$ are closed and $\Omega_{r}^{-}=B_{1} \cup \ldots \cup B_{n}$. Define, for $0<\mu<\bar{\mu}$, the deformation

$$
g_{j}:[0,1] \times B_{j} \rightarrow \Omega_{r}^{+}, \quad(t, y) \mapsto g_{j}(t, y)=\beta\left(h_{j}\left(t, \gamma_{\mu}(y)\right)\right) .
$$

By Lemma 4.7, the deformation $g_{j}$ is well defined, and from (4.10) and (4.11)

$$
\begin{array}{ll}
g_{j}(0, y)=\beta\left(h_{j}\left(0, \gamma_{\mu}(y)\right)\right)=\beta\left(\gamma_{\mu}(y)\right)=y, & \text { for all } y \in B_{j}, \\
g_{j}(1, y)=\beta\left(h_{j}\left(1, \gamma_{\mu}(y)\right)\right)=\beta\left(w_{j}\right), & \text { for all } y \in B_{j} .
\end{array}
$$

Hence, the sets $B_{j}$ are contractible in $\Omega_{r}^{+}$, and so

$$
\operatorname{cat}_{\Omega}(\Omega)=\operatorname{cat}_{\Omega_{r}^{+}}\left(\Omega_{r}^{-}\right) \leq n=\operatorname{cat}_{I_{\mu}^{m(\mu, r)}}\left(I_{\mu}^{m(\mu, r)}\right) .
$$

Proof of Theorem 1.1 (completed). By Lemmas 3.4 and 4.3, for $c \leq$ $m(\mu, \Omega) \leq m(\mu, r)<S,\left.I_{\mu}\right|_{V}$ satisfies the (PS) $)_{c}$-condition. By Theorem 4.1, with $d=m(\mu, r)$, it follows that $I_{\mu}^{m(\mu, r)}$ has at least $\operatorname{cat}_{I_{\mu}^{m(\mu, r)}\left(I_{\mu}^{m(\mu, r)}\right) \text { critical }}$ points of $\left.I_{\mu}\right|_{V}$. Then, by Lemma 4.10 , for $0<\mu<\bar{\mu}$, we have that $\left.I_{\mu}\right|_{V}$ has at least $n=\operatorname{cat}_{\Omega}(\Omega)$ different critical points, say $v_{1}, \ldots, v_{n} \in V$.

For each $j=1, \ldots, n$, there exists $\lambda_{j} \in \mathbb{R}$ such that $v_{j}$ satisfies

$$
\begin{cases}\Delta^{2} v_{j}=\mu\left(v_{j}^{+}\right)+\lambda_{j}\left(v_{j}^{+}\right)^{2 *-1}, & \text { in } \Omega, \\ v_{j}, \Delta v_{j}=0 & \text { on } \partial \Omega .\end{cases}
$$

Since $v_{j} \in V$ we have $v_{j} \neq 0$, and

$$
\lambda_{j}=\lambda_{j} \int_{\Omega}\left(v_{j}^{+}\right)^{2 *} d x=I_{\mu}\left(v_{j}\right)=\int_{\Omega}\left[\left|\Delta v_{j}\right|^{2}-\mu\left(v_{j}^{+}\right)^{2}\right] d x>0 .
$$

Hence, for each $j=1, \ldots, n$, we have that $u_{j}:=\lambda_{j}^{1 /\left(2_{*}-2\right)} v_{j}$ is a nontrivial solution of

$$
\begin{cases}\Delta^{2} u=\mu u^{+}+\left(u^{+}\right)^{2_{*}-1} & \text { in } \Omega, \\ u, \Delta u=0 & \text { on } \partial \Omega,\end{cases}
$$

that is, $u_{j}$ is a critical point of $I$. Since $v_{j} \neq v_{i}$ if $j \neq i$, it follows that $u_{j} \neq u_{i}$ if $j \neq i$. Then, we apply Lemma 2.2 to end this proof. 


\section{Appendix A. Proof of Lemma 3.1}

Proof. Particular case: Assume first $u=0$.

For every $h \in \mathcal{C}_{c}^{\infty}\left(\mathbb{R}^{N}\right)$, we infer from (2.2) that

$$
\left(\int_{\mathbb{R}^{N}}\left|h u_{n}\right|^{2 *} d x\right)^{2 / 2_{*}} \leq S^{-1} \int_{\mathbb{R}^{N}}\left|\Delta\left(h u_{n}\right)\right|^{2} d x .
$$

Using (3.2) and (3.3) we get

$$
\left(\int_{\mathbb{R}^{N}}|h|^{2_{*}}\left|u_{n}\right|^{2_{*}} d x\right)^{2 / 2_{*}} \rightarrow\left(\int_{\mathbb{R}^{N}}|h|^{2_{*}} d \nu\right)^{2 / 2_{*}}
$$

and

$$
\int_{\mathbb{R}^{N}}|h|^{2}\left|\Delta u_{n}\right|^{2} d x \rightarrow \int_{\mathbb{R}^{N}}|h|^{2} d \lambda .
$$

Note that

$$
\Delta\left(h u_{n}\right)-h \Delta u_{n}=u_{n} \Delta h+2 \nabla h . \nabla u_{n} .
$$

We have

$$
\left|u_{n} \Delta h\right|_{2}^{2}=\int_{B_{R}(0)}|\Delta h|^{2}\left|u_{n}\right|^{2} d x \leq C \int_{B_{R}(0)}\left|u_{n}\right|^{2} d x,
$$

where $R>0$ is such that $\operatorname{supp}(h) \subset \bar{B}_{R}(0)$ and $C=\max _{\bar{B}_{R}(0)}|\Delta h|^{2}$. Then

$$
\left|u_{n} \Delta h\right|_{2}^{2} \rightarrow 0,
$$

because $u_{n} \rightarrow 0$ in $L_{\text {loc }}^{2}\left(\mathbb{R}^{N}\right)$. We also have

$$
\left|\nabla h . \nabla u_{n}\right|_{2}^{2} \leq \int_{B_{R}(0)}|\nabla h|^{2}\left|\nabla u_{n}\right|^{2} d x \leq \bar{C} \int_{B_{R}(0)}\left|\nabla u_{n}\right|^{2} d x,
$$

where $\bar{C}=\max _{\bar{B}_{R}(0)}|\nabla h|^{2}$, and consequently

$$
\left|\nabla h . \nabla u_{n}\right|_{2}^{2} \rightarrow 0,
$$

because $\nabla u_{n} \rightarrow 0$ in $\left[L_{\text {loc }}^{2}\left(\mathbb{R}^{N}\right)\right]^{N}$. From (A.4)-(A.6) follows that

$$
\begin{aligned}
\|\left.\Delta\left(h u_{n}\right)\right|_{2}-\left|h \Delta u_{n}\right|_{2} \mid & \leq\left|\Delta\left(h u_{n}\right)-h \Delta u_{n}\right|_{2} \\
& =\left|u_{n} \Delta h+2 \nabla h . \nabla u_{n}\right|_{2} \leq\left|u_{n} \Delta h\right|_{2}+2\left|\nabla h . \nabla u_{n}\right|_{2} \rightarrow 0,
\end{aligned}
$$

that is,

$$
\lim _{n \rightarrow \infty} \int_{\mathbb{R}^{N}}\left|\Delta\left(h u_{n}\right)\right|^{2} d x=\lim _{n \rightarrow \infty} \int_{\mathbb{R}^{N}}\left|h \Delta u_{n}\right|^{2} d x=\int_{\mathbb{R}^{N}}|h|^{2} d \lambda .
$$

Hence, from (A.1)-(A.2) we get

$$
\left(\int_{\mathbb{R}^{N}}|h|^{2_{*}} d \nu\right)^{2 / 2_{*}} \leq S^{-1} \int_{\mathbb{R}^{N}}|h|^{2} d \lambda .
$$


Taking now the sequence $\left(h_{n}\right) \subset \mathcal{C}_{c}^{\infty}\left(\mathbb{R}^{N}\right)$ such that

$$
h_{n} \equiv 1 \quad \text { in } B_{n}(0), \quad \operatorname{supp}\left(h_{n}\right) \subset B_{n+1}(0), \quad 0 \leq h_{n} \leq 1,
$$

it follows by dominated convergence theorem that

$$
\lim _{n \rightarrow \infty} \int_{\mathbb{R}^{N}}\left|h_{n}\right|^{2 *} d \nu=\int_{\mathbb{R}^{N}} 1 d \nu=\|\nu\| \quad \text { and } \quad \lim _{n \rightarrow \infty} \int_{\mathbb{R}^{N}}\left|h_{n}\right|^{2} d \lambda=\int_{\mathbb{R}^{N}} 1 d \lambda=\|\lambda\| .
$$

Then we obtain (3.5) using $\left(h_{n}\right)$ in (A.7) and taking $n \rightarrow \infty$.

Now we proceed to prove (3.6). Fix $R>0$ and let $\psi_{R} \in \mathcal{C}^{\infty}\left(\mathbb{R}^{N}\right)$ be such that $\psi_{R}(x)=1$ for $|x| \geq R+1, \psi_{R}(x)=0$ for $|x| \leq R$ and $0 \leq \psi_{R} \leq 1$ on $\mathbb{R}^{N}$. By the Sobolev inequality, we have

$$
\varlimsup_{n \rightarrow \infty}\left(\int_{\mathbb{R}^{N}}\left|\psi_{R} u_{n}\right|^{2 *} d x\right)^{2 / 2_{*}} \leq S^{-1} \varlimsup_{n \rightarrow \infty} \int_{\mathbb{R}^{N}}\left|\Delta\left(\psi_{R} u_{n}\right)\right|^{2} d x .
$$

We have

$$
0 \leq \int_{\mathbb{R}^{N}}\left|u_{n} \Delta \psi_{R}\right|^{2} d x \leq \int_{|x| \leq R+1}\left|\Delta \psi_{R}\right|^{2}\left|u_{n}\right|^{2} d x \leq C_{R} \int_{|x| \leq R+1}\left|u_{n}\right|^{2} d x,
$$

where $C_{R}=\max _{\bar{B}_{R+1}(0)}\left|\Delta \psi_{R}\right|^{2}$, and

$$
0 \leq \int_{\mathbb{R}^{N}}\left|\nabla \psi_{R} \cdot \nabla u_{n}\right|^{2} d x \leq \int_{|x| \leq R+1}\left|\nabla \psi_{R}\right|^{2}\left|\nabla u_{n}\right|^{2} d x \leq D_{R} \int_{|x| \leq R+1}\left|\nabla u_{n}\right|^{2} d x,
$$

where $D_{R}=\max _{\bar{B}_{R+1}(0)}\left|\nabla \psi_{R}\right|^{2}$. Thus

$$
\|\left.\Delta\left(\psi_{R} u_{n}\right)\right|_{2}-\left.\left|\psi_{R} \Delta u_{n}\right|_{2}|\leq| u_{n} \psi_{R}\right|_{2}+\left|2 \nabla \psi_{R} . \nabla u_{n}\right|_{2} \rightarrow 0,
$$

because $u_{n}, \nabla u_{n} \rightarrow 0$ in $L_{\mathrm{loc}}^{2}\left(\mathbb{R}^{N}\right),\left[L_{\mathrm{loc}}^{2}\left(\mathbb{R}^{N}\right)\right]^{N}$, respectively. From (A.8) we conclude

$$
\varlimsup_{n \rightarrow \infty}\left(\int_{\mathbb{R}^{N}} \psi_{R}^{2_{*}}\left|u_{n}\right|^{2 *}, d x\right)^{2 / 2_{*}} \leq S^{-1} \varlimsup_{n \rightarrow \infty} \int_{\mathbb{R}^{N}} \psi_{R}^{2}\left|\Delta u_{n}\right|^{2} d x .
$$

On the another hand, we have

$$
\int_{\mathbb{R}^{N}}\left|\Delta u_{n}\right|^{2} \psi_{R}^{2} d x=\int_{|x| \geq R}\left|\Delta u_{n}\right|^{2} \psi_{R}^{2} d x \leq \int_{|x| \geq R}\left|\Delta u_{n}\right|^{2} d x
$$

and

$$
\int_{|x| \geq R+1}\left|u_{n}\right|^{2_{*}} d x=\int_{|x| \geq R+1}\left|u_{n}\right|^{2_{*}} \psi_{R}^{2_{*}} d x \leq \int_{\mathbb{R}^{N}}\left|u_{n}\right|^{2_{*}} \psi_{R}^{2_{*}} d x
$$

and from (A.9) follows that

$$
\begin{aligned}
& \nu_{\infty}^{2 / 2_{*}}=\lim _{R \rightarrow \infty} \varlimsup_{n \rightarrow \infty}\left(\int_{|x| \geq R+1}\left|u_{n}\right|^{2_{*}} d x\right)^{2 / 2_{*}} \\
& \leq S^{-1} \lim _{R \rightarrow \infty} \varlimsup_{n \rightarrow \infty}\left(\int_{|x| \geq R}\left|\Delta u_{n}\right|^{2} d x\right)=S^{-1} \lambda_{\infty},
\end{aligned}
$$

which proves (3.6). 
Assume moreover, that $\|\nu\|^{2 / 2_{*}}=S^{-1}\|\mu\|$. We will show that $\lambda$ and $\nu$ are concentrated at a common single point. Given $h \in \mathcal{C}_{c}^{\infty}\left(\mathbb{R}^{N}\right)$ we have, from (A.7),

$$
\left(\int_{\mathbb{R}^{N}}|h|^{2 *} d \nu\right)^{1 / 2 *} \leq S^{-1 / 2}\left(\int_{\mathbb{R}^{N}}|h|^{2} d \lambda\right)^{1 / 2}
$$

and from Hölder inequality we get

$$
\int_{\mathbb{R}^{N}}|h|^{2 *} d \nu \leq S^{-2 * / 2}\|\lambda\|^{4 /(N-4)} \int_{\mathbb{R}^{N}}|h|^{2_{*}^{*}} d \lambda, \quad \text { for all } h \in \mathcal{C}_{c}^{\infty}\left(\mathbb{R}^{N}\right),
$$

which implies

$$
\nu(\Omega) \leq S^{-2 * / 2}\|\lambda\|^{4 /(N-4)} \lambda(\Omega), \quad \text { for all } \Omega \subset \mathbb{R}^{N} \text { measurable. }
$$

We prove now that $\nu(\Omega)=S^{-2 * / 2}\|\lambda\|^{4 /(N-4)} \lambda(\Omega)$, for all $\Omega \subset \mathbb{R}^{N}$ measurable. Assume that there exists $\Omega_{0} \subset \mathbb{R}^{N}$ such that $\nu\left(\Omega_{0}\right)<S^{-2 * / 2}\|\lambda\|^{4 /(N-4)} \lambda\left(\Omega_{0}\right)$. By hypothesis, $\|\nu\|^{2 / 2_{*}}=S^{-1}\|\lambda\|$, which implies

$$
\nu\left(\mathbb{R}^{N}\right)=S^{-2 * / 2}\|\lambda\|^{4 /(N-4)} \lambda\left(\mathbb{R}^{N}\right)
$$

Note that

$$
\begin{aligned}
\nu\left(\mathbb{R}^{N}\right) & =\nu\left(\Omega_{0}\right)+\nu\left(\mathbb{R}^{N} \backslash \Omega_{0}\right) \\
& <S^{-2_{*} / 2}\|\lambda\|^{4 /(N-4)} \lambda\left(\Omega_{0}\right)+S^{-2_{*} / 2}\|\lambda\|^{4 /(N-4)} \lambda\left(\mathbb{R}^{N} \backslash \Omega_{0}\right) \\
& =S^{-2 * / 2}\|\lambda\|^{4 /(N-4)}\left[\lambda\left(\Omega_{0}\right)+\lambda\left(\mathbb{R}^{N} \backslash \Omega_{0}\right)\right]=S^{-2 * / 2}\|\lambda\|^{4 /(N-4)} \lambda\left(\mathbb{R}^{N}\right)
\end{aligned}
$$

which contradicts (A.12). It follows from (A.10), $\nu(\Omega)=S^{-2_{*} / 2}\|\lambda\|^{4 /(N-4)} \lambda(\Omega)$ and $\|\nu\|^{2 / 2_{*}}=S^{-1}\|\lambda\|$ that

$$
\left(\int_{\mathbb{R}^{N}}|h|^{2_{*}} d \nu\right)^{1 / 2_{*}}\|\nu\|^{2 / N} \leq\left(\int_{\mathbb{R}^{N}}|h|^{2} d \nu\right)^{1 / 2}, \quad \text { for all } h \in \mathcal{C}_{c}^{\infty}\left(\mathbb{R}^{N}\right) .
$$

Then, for each open set $\Omega \subset \mathbb{R}^{N}$,

$$
\nu(\Omega)^{1 / 2 *} \nu\left(\mathbb{R}^{N}\right)^{2 / N} \leq \nu(\Omega)^{1 / 2} .
$$

Since $1 / 2-1 / 2_{*}=2 / N$, we have

$$
\nu(\Omega)=0 \quad \text { or } \quad \nu(\Omega) \geq \nu\left(\mathbb{R}^{N}\right), \quad \text { for any open set } \Omega \subset \mathbb{R}^{N} .
$$

Hence, $\nu$ is concentrated at a single point, which is the same point where $\lambda$ concentrates, because $\nu=S^{-2 * / 2}\|\lambda\|^{4 /(N-4)} \lambda$.

General case: $u$ is not necessarily zero and we prove (3.5)-(3.8).

Write $v_{n}:=u_{n}-u$. So, $v_{n} \rightarrow 0$ in $\mathcal{D}^{2,2}\left(\mathbb{R}^{N}\right),\left|\Delta v_{n}\right|^{2} \stackrel{*}{\rightarrow} \lambda$ and $\left|v_{n}\right|^{2 *} \stackrel{*}{\rightarrow} \nu$ in the sense of measures on $\mathbb{R}^{N}$, and $v_{n} \rightarrow 0$ almost everywhere on $\mathbb{R}^{N}$, and thus, from the previous case, (3.5) holds. 
We have

$$
\begin{aligned}
\int_{|x| \geq R}\left|\Delta u_{n}\right|^{2} d x & =\int_{|x| \geq R}\left|\Delta v_{n}+\Delta u\right|^{2} d x \\
& =\int_{|x| \geq R}\left|\Delta v_{n}\right|^{2} d x+2 \int_{|x| \geq R} \Delta v_{n} \Delta u d x+\int_{|x| \geq R}|\Delta u|^{2} d x
\end{aligned}
$$

which implies

$$
\begin{aligned}
& \varlimsup_{n \rightarrow \infty} \int_{|x| \geq R}\left|\Delta u_{n}\right|^{2} d x \\
& \quad=\varlimsup_{n \rightarrow \infty} \int_{|x| \geq R}\left|\Delta v_{n}\right|^{2} d x+2 \varlimsup_{n \rightarrow \infty} \int_{|x| \geq R} \Delta v_{n} \Delta u d x+\int_{|x| \geq R}|\Delta u|^{2} d x
\end{aligned}
$$

and, since $v_{n} \rightarrow 0$ in $\mathcal{D}^{2,2}\left(\mathbb{R}^{N}\right)$, we conclude that

$$
\varlimsup_{n \rightarrow \infty} \int_{|x| \geq R}\left|\Delta u_{n}\right|^{2} d x=\varlimsup_{n \rightarrow \infty} \int_{|x| \geq R}\left|\Delta v_{n}\right|^{2} d x+\int_{|x| \geq R}|\Delta u|^{2} d x .
$$

So, (A.13) implies that

$$
\lambda_{\infty}=\lim _{R \rightarrow \infty} \varlimsup_{n \rightarrow \infty} \int_{|x| \geq R}\left|\Delta u_{n}\right|^{2} d x=\lim _{R \rightarrow \infty} \varlimsup_{n \rightarrow \infty} \int_{|x| \geq R}\left|\Delta v_{n}\right|^{2} d x .
$$

By the Brézis-Lieb lemma [7],

$$
\int_{|x| \geq R}|u|^{2 *} d x=\lim _{n \rightarrow \infty}\left(\int_{|x| \geq R}\left|u_{n}\right|^{2 *} d x-\int_{|x| \geq R}\left|v_{n}\right|^{2 *} d x\right),
$$

and therefore

$$
\nu_{\infty}=\lim _{R \rightarrow \infty} \varlimsup_{n \rightarrow \infty} \int_{|x| \geq R}\left|u_{n}\right|^{2 *} d x=\lim _{R \rightarrow \infty} \varlimsup_{n \rightarrow \infty} \int_{|x| \geq R}\left|v_{n}\right|^{2 *} d x .
$$

From the previous particular case, it follows (3.6).

Now we proceed to prove (3.7). First we prove that

$$
\left|\Delta u_{n}\right|^{2} \stackrel{*}{\rightarrow} \lambda+|\Delta u|^{2} .
$$

Indeed, from the identity $\left|\Delta u_{n}\right|^{2}=\left|\Delta v_{n}+\Delta u\right|^{2}=\left|\Delta v_{n}\right|^{2}+2 \Delta v_{n} \Delta u+|\Delta u|^{2}$, we have

$$
\int_{\mathbb{R}^{N}} \varphi\left|\Delta u_{n}\right|^{2} d x=\int_{\mathbb{R}^{N}} \varphi\left|\Delta v_{n}\right|^{2} d x+2 \int_{\mathbb{R}^{N}} \Delta v_{n} \Delta u \varphi d x+\int_{\mathbb{R}^{N}} \varphi|\Delta u|^{2} d x,
$$

for all $\varphi \in \mathcal{C}_{0}\left(\mathbb{R}^{N}\right)$. Since $v_{n} \rightarrow 0$ in $\mathcal{D}^{2,2}\left(\mathbb{R}^{N}\right)$ and $\left|\Delta v_{n}\right|^{2} \stackrel{*}{\rightarrow} \lambda$ we obtain

$$
\lim _{n \rightarrow \infty} \int_{\mathbb{R}^{N}} \varphi\left|\Delta u_{n}\right|^{2} d x=\int_{\mathbb{R}^{N}} \varphi d \lambda+\int_{\mathbb{R}^{N}} \varphi|\Delta u|^{2} d x
$$

for all $\varphi \in \mathcal{C}_{0}\left(\mathbb{R}^{N}\right)$, which is precisely (A.14). 
Fix $R>0$ and let $\psi_{R} \in \mathcal{C}^{\infty}\left(\mathbb{R}^{N}\right)$ be such that $\psi_{R}(x)=1$ for $|x| \geq R+1$, $\psi_{R}(x)=0$ for $|x| \leq R$ and $0 \leq \psi_{R} \leq 1$ on $\mathbb{R}^{N}$. From (A.14) we have

$$
\begin{aligned}
\varlimsup_{n \rightarrow \infty} \int_{\mathbb{R}^{N}}\left|\Delta u_{n}\right|^{2} d x \\
\quad=\varlimsup_{n \rightarrow \infty} \int_{\mathbb{R}^{N}} \psi_{R}\left|\Delta u_{n}\right|^{2} d x+\int_{\mathbb{R}^{N}}\left(1-\psi_{R}\right) d \lambda+\int_{\mathbb{R}^{N}}\left(1-\psi_{R}\right)|\Delta u|^{2} d x .
\end{aligned}
$$

Taking now $R \rightarrow \infty$, it follows from the dominated convergence theorem that

$$
\lim _{R \rightarrow \infty} \varlimsup_{n \rightarrow \infty} \int_{\mathbb{R}^{N}}\left|\Delta u_{n}\right|^{2} d x=\lambda_{\infty}+\int_{\mathbb{R}^{N}} 1 d \lambda+\int_{\mathbb{R}^{N}}|\Delta u|^{2} d x
$$

and thus

$$
\varlimsup_{n \rightarrow \infty}\left|\Delta u_{n}\right|_{2}^{2}=|\Delta u|_{2}^{2}+\|\lambda\|+\lambda_{\infty},
$$

which is precisely (3.7).

To prove (3.8), first observe that

$$
\left|u_{n}\right|^{2 *} \stackrel{*}{\rightarrow} \nu+|u|^{2 *} .
$$

Indeed, for any $f \in \mathcal{C}_{0}\left(\mathbb{R}^{N}\right)$ we have, from the Brézis-Lieb [7] lemma applied to $f^{+}$and $f^{-}$,

$$
\int_{\mathbb{R}^{N}} f|u|^{2_{*}} d x=\lim _{n \rightarrow \infty}\left(\int_{\mathbb{R}^{N}} f\left|u_{n}\right|^{2_{*}} d x-\int_{\mathbb{R}^{N}} f\left|v_{n}\right|^{2_{*}} d x\right),
$$

from where (A.15) follows since $\left|v_{n}\right|^{2 *} \stackrel{*}{\rightarrow} \nu$.

Fix $R>0$ and let $\psi_{R} \in \mathcal{C}^{\infty}\left(\mathbb{R}^{N}\right)$ be such that $\psi_{R}(x)=1$ for $|x| \geq R+1$, $\psi_{R}(x)=0$ for $|x| \leq R$ and $0 \leq \psi_{R} \leq 1$ on $\mathbb{R}^{N}$. Then

$\varlimsup_{n \rightarrow \infty} \int_{\mathbb{R}^{N}}\left|u_{n}\right|^{2_{*}} d x=\varlimsup_{n \rightarrow \infty} \int_{\mathbb{R}^{N}} \psi_{R}\left|u_{n}\right|^{2_{*}} d x+\int_{\mathbb{R}^{N}}\left(1-\psi_{R}\right) d \nu+\int_{\mathbb{R}^{N}}\left(1-\psi_{R}\right)|u|^{2_{*}} d x$.

Taking $R \rightarrow \infty$, it follows from the dominated convergence theorem that

$$
\lim _{R \rightarrow \infty} \varlimsup_{n \rightarrow \infty} \int_{\mathbb{R}^{N}}\left|u_{n}\right|^{2_{*}} d x=\nu_{\infty}+\int_{\mathbb{R}^{N}} 1 d \nu+\int_{\mathbb{R}^{N}}|u|^{2_{*}} d x
$$

and thus

$$
\varlimsup_{n \rightarrow \infty}\left|u_{n}\right|_{2_{*}}^{2_{*}}=|u|_{2_{*}}^{2_{*}}+\|\nu\|+\nu_{\infty} .
$$

\section{Appendix B. Proof of Lemma 4.2}

Proof. Without loss of generality, suppose $0 \in \Omega$. Let $\xi \in \mathcal{C}_{c}^{\infty}\left(\mathbb{R}^{N}\right)$ be a function such that $0 \leq \xi(x) \leq 1$, for all $x \in \mathbb{R}^{N}, \xi \equiv 1$ in $B(0, \rho / 2), \xi \equiv 0$ in $B(0, \rho)^{c}$, and $B(0, \rho) \subset \subset \Omega, \rho>0$. Set

$$
U_{\delta}(x):=\xi(x) \psi_{\delta}(x), \quad x \in \mathbb{R}^{N}, 0<\delta<\rho,
$$

where $\psi_{\delta}=S^{(4-N) / 8} \varphi_{\delta}$ and $\varphi_{\delta}(x)=\varphi_{\delta, 0}(x)$ is given by (2.3). Then

$$
\int_{\mathbb{R}^{N}}\left|\Delta \psi_{\delta}\right|^{2} d x=S \quad \text { and } \quad \int_{\mathbb{R}^{N}}\left|\psi_{\delta}\right|^{2 *} d x=1,
$$


and, see $[10,(6.4)$ and (6.3)] respectively, we have

$$
\begin{aligned}
\left|\Delta U_{\delta}\right|_{2, \Omega}^{2} & =S+O\left(\delta^{N-4}\right), \\
\left|U_{\delta}\right|_{2_{*}, \Omega}^{2_{*}} & =1+O\left(\delta^{N}\right) .
\end{aligned}
$$

In order to get (4.1), we will estimate $\left|U_{\delta}\right|_{2, \Omega}^{2}$. We have

$\left|U_{\delta}\right|_{2, \Omega}^{2}=\int_{\Omega}\left|\xi(x) \psi_{\delta}(x)\right|^{2} d x=\int_{B(0, \rho)}\left|\psi_{\delta}(x)\right|^{2} d x+\int_{B(0, \rho)}\left[|\xi(x)|^{2}-1\right]\left|\psi_{\delta}(x)\right|^{2} d x$.

Note that

$$
\begin{aligned}
& \left.\int_{B(0, \rho)}|| \xi(x)\right|^{2}-\left.1|| \psi_{\delta}(x)\right|^{2} d x \\
& =\left.\int_{B(0, \rho) \backslash B(0, \rho / 2)}|| \xi(x)\right|^{2}-\left.1|| \psi_{\delta}(x)\right|^{2} d x \leq \int_{B(0, \rho) \backslash B(0, \rho / 2)}\left|\psi_{\delta}(x)\right|^{2} d x \\
& =\int_{B(0, \rho) \backslash B(0, \rho / 2)} \frac{C \delta^{N-4}}{\left(\delta^{2}+|x|^{2}\right)^{N-4}} d x \leq \int_{B(0, \rho) \backslash B(0, \rho / 2)} \frac{C \delta^{N-4}}{|x|^{2(N-4)}} d x=O\left(\delta^{N-4}\right) .
\end{aligned}
$$

So, we obtain

$$
\int_{\Omega}\left|U_{\delta}(x)\right|^{2} d x=\int_{B(0, \rho)}\left|\psi_{\delta}(x)\right|^{2} d x+O\left(\delta^{N-4}\right) .
$$

Now,

$$
\int_{B(0, \rho)}\left|\psi_{\delta}(x)\right|^{2} d x=\int_{B(0, \delta)}\left|\psi_{\delta}(x)\right|^{2} d x+\int_{\delta<|x|<\rho}\left|\psi_{\delta}(x)\right|^{2} d x .
$$

Note that

$$
\begin{aligned}
\int_{B(0, \delta)}\left|\psi_{\delta}(x)\right|^{2} d x & =\int_{B(0, \delta)} \frac{C \delta^{N-4}}{\left(\delta^{2}+|x|^{2}\right)^{N-4}} d x \\
& \geq \int_{B(0, \delta)} \frac{C \delta^{N-4}}{\left(2 \delta^{2}\right)^{N-4}} d x=C \delta^{4},
\end{aligned}
$$

and

$$
\begin{array}{r}
\int_{\delta<|x|<\rho}\left|\psi_{\delta}(x)\right|^{2} d x=\int_{\delta<|x|<\rho} \frac{C \delta^{N-4}}{\left(\delta^{2}+|x|^{2}\right)^{N-4}} d x \geq \int_{\delta<|x|<\rho} \frac{C \delta^{N-4}}{\left(2|x|^{2}\right)^{N-4}} d x \\
=C \delta^{N-4} \int_{\delta<|x|<\rho} \frac{1}{|x|^{2(N-4)}} d x=C \delta^{N-4} \int_{\delta}^{\rho} \int_{S_{r}} \frac{1}{r^{2(N-4)}} d S d r,
\end{array}
$$

which implies

$$
\int_{\delta<|x|<\rho}\left|\psi_{\delta}(x)\right|^{2} d x \geq C \delta^{N-4} \begin{cases}\left.\log r\right|_{\delta} ^{\rho} & \text { if } N=8 \\ -\left.\frac{1}{N-8} \frac{1}{r^{N-8}}\right|_{\delta} ^{\rho} & \text { if } N>8 .\end{cases}
$$

Finally, combining (B.3)-(B.6), we conclude that

$$
\left|U_{\delta}\right|_{2, \Omega}^{2} \geq \begin{cases}C \delta^{4}|\log \delta|+O\left(\delta^{4}\right) & \text { if } N=8, \\ C \delta^{4}+O\left(\delta^{N-4}\right) & \text { if } N>8 .\end{cases}
$$


Then, from (B.1), (B.2) and (B.7), there exists a constant $C=C(N)>0$ such that

$$
\frac{\left|\Delta U_{\delta}\right|_{2}^{2}-\mu\left|U_{\delta}\right|_{2}^{2}}{\left|U_{\delta}\right|_{2_{*}}^{2}} \leq\left\{\begin{array}{ll}
S-\mu C \delta^{4}|\log \delta|+O\left(\delta^{4}\right), & N=8, \\
S-\mu C \delta^{4}+O\left(\delta^{N-4}\right), & N>8,
\end{array}\right\}<S
$$

for $N \geq 8$ and $\delta>0$ small.

\section{REFERENCES}

[1] W. ABdeLHEDI, On a fourth-order elliptic equation involving the critical Sobolev exponent: The effect of the graph topology, Nonlinear Anal. 82 (2013), 82-99.

[2] S. Agmon, A. Douglis and L. Nirenberg, Estimates near the boundary for solutions of elliptic partial differential equations satisfying general boundary conditions I, Comm. Pure Appl. Math. 12 (1959), 623-727.

[3] A. Alvino, P.-L. Lions And G. Trombetti, A remark on comparison results via symmetrization, Proc. Roy. Soc. Edinburgh Sect. A 102 (1986), no. 1-2, 37-48.

[4] A.K. Ben-Naoum, C. Troestler And M. Willem, Extrema problems with critical Sobolev exponents on unbounded domains, Nonlinear Anal. 26 (1996), no. 4, 823-833.

[5] G. Bianchi, J. Chabrowski And A. Szulkin, On symmetric solutions of an elliptic equation with a nonlinearity involving critical Sobolev exponent, Nonlinear Anal. 25 (1995), no. $1,41-59$.

[6] D. Bonheure, E. Moreira dos Santos And M. Ramos, Ground state and non-ground state solutions of some strongly coupled elliptic systems, Trans. Amer. Math. Soc. 364 (2012), no. 1, 447-491.

[7] H. BRÉzIS AND E. LieB, A relation between pointwise convergence of functions and convergence of functionals, Proc. Amer. Math. Soc. 88 (1983), no. 3, 486-490.

[8] sc H. Brézis, L. Nirenberg, Positive solutions of nonlinear elliptic equations involving critical Sobolev exponents, Comm. Pure Appl. Math. 36 (1983), 437-477.

[9] P. Clément, P. Felmer and E. Mitidieri, Homoclinic orbits for a class of infinite dimensional hamiltonian systems, Ann. Scuola Norm. Sup. Pisa Cl. Sci. (4) 24 (1997), no. 2, 367-393.

[10] E.M. Dos SAntos, Positive solutions for a fourth-order quasilinear equation with critical Sobolev exponent, Commun. Contemp. Math. 12 (2010), no. 1, 1-33.

[11] K. El Mehdi And A. Selmi, Concentration and multiplicity of solutions for a fourth-order equation with critical nonlinearity, Nonlinear Anal. 64 (2006), no. 3, 417-439.

[12] A. Ferrero, F. Gazzola and T. Weth, Positivity, symmetry and uniqueness for minimizers of second-order Sobolev inequalities,Ann. Mat. Pura Appl. (4) 186 (2007), no. 4, $565-578$.

[13] F. Gazzola, H.-C. Grunau and M. Squassina, Existence and nonexistence results for critical growth biharmonic elliptic equations, Calc. Var. Partial Differential Equations 18 (2003), no. 2, 117-143.

[14] F. Gazzola, H.-C. Grunau and G. Sweers, Polyharmonic Boundary Value Problems. Positivity Preserving and Nonlinear Higher Order Elliptic Equations in Bounded Domains, Lecture Notes in Mathematics 1991. Springer-Verlag (2010).

[15] D. Gilbarg and N.S. Trudinger, Elliptic Partial Differential Equations of Second Order, Springer-Verlag, 1977.

[16] O. Kavian, Introduction à la théorie des points critiques: et applications aux problèmes elliptiques, Math. Appl. 13, Springer-Verlag (1993). 
[17] M. LAzzo, Solutions positives multiples pour une équation elliptique non linaire avec l'exposant critique de Sobolev, C.R. Acad. Sci. Paris Sér. I Math. 314 (1992), no. 1, 61-64.

[18] P.-L. Lions, The concentration-compactness principle in the calculus of variations. The limit case I, Rev. Mat. Iberoamericana 1 (1985), no. 1, 145-201.

[19] L. Lusternik And L. Schnirelmann, Méthodes topologiques dans les problèmes variationnels, Hermann, Paris, 1934.

[20] P.J. McKenna And W. ReicheL, Radial solutions of singular nonlinear biharmonic equations and applications to conformal geometry, Electron. J. Differential Equations (2003), no. 37,13 pp.

[21] W.M. NI, A nonlinear Dirichlet problem on the unit ball and its applications, Indiana Univ. Math. J. 31 (1982), no. 6, 801-807.

[22] S.I. PohožAev, On the eigenfunctions of the equation $\Delta u+\lambda f(u)=0$, Dokl. Akad. Nauk SSSR 165 (1965), 36-39.

[23] O. ReY, A multiplicity result for a variational problem with lack of compactness, Nonlinear Anal. 13 (1989), no. 10, 1241-1249.

[24] _ Proof of two conjectures of H. Brézis and L.A. Peletier, Manuscripta Math. 65 (1989), no. 1, 19-37.

[25] _ . The role of the Green's function in a nonlinear elliptic equation involving the critical Sobolev exponent, J. Funct. Anal. 89 (1990), no. 1, 1-52.

[26] R.C.A.M. VAN DER Vorst, Best constant for the embedding for the space $H^{2} \cap H_{0}^{1}(\Omega)$ into $L^{2 N /(N-4)}(\Omega)$, Differ. Integral Equations 6 (1993), 259-276.

[27] _ Fourth order elliptic equations with critical growth, C.R. Acad. Sci. Paris Serr. I Math. 320 (1995), no. 3, 295-299.

[28] M. Willem, Minimax theorems, Progress in Nonlinear Differential Equations and their Applications, 24. Birkhäuser, 1996.

JÉSSyCa LANGe Ferreira Melo

Departamento de Matemática

Univerisdade Federal de Viçosa

CEP 36570-000, Viçosa, MG, BRAZIL

E-mail address: jessycalange@gmail.com

Ederson Moreira dos Santos

Instituto de Ciências Matemáticas e de Computação

Universidade de São Paulo

C.P. 668, CEP 13560-970 São Carlos SP, BRAZIL

E-mail address: ederson@icmc.usp.br 